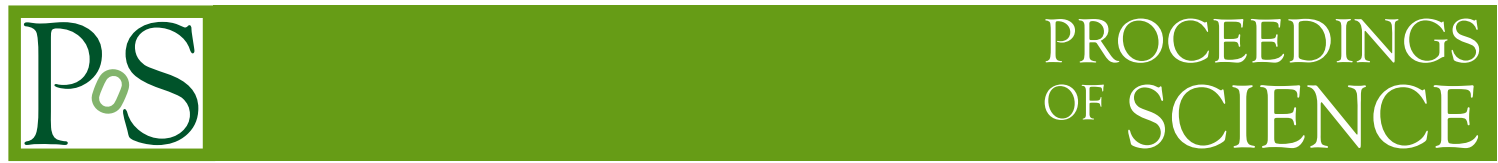

\title{
Null-controllability of some reaction-diffusion systems with one control force
}

\author{
Assia Benabdallah * \\ Laboratoire d'Analyse, Topologie, Probabilités \\ CNRS / Université de Provence \\ E-mail.assiadcmi.univ-mrs.fr \\ Farid Ammar Khodja \\ Université de Franche-Comté \\ E-mail: ammaramath.univ-fcomte.fr

\section{Cédric Dupaix} \\ Université de Franche-Comté \\ E-mail:dupaix@math.univ-fcomte.fr
}

This work is concerned with the null-controllability of semilinear parabolic systems by a single control force acting on a subdomain.

Control Systems: Theory, Numerics and Applications

30 March - 1 April 2005

Rome

\footnotetext{
* Speaker.
} 


\section{Introduction}

We consider a general reaction-diffusion system which arises in mathematical biology :

$$
\begin{aligned}
& \psi_{t}=\Delta \psi+f_{1}(\psi, w), \text { in } Q_{T}=\Omega \times(0, T) \\
& w_{t}=\Delta w+f_{2}(\psi, w)+\chi_{\omega} g, \text { in } Q_{T} \\
& \psi=w=0 \quad \text { on } \Sigma_{T}=\partial \Omega \times(0, T) \\
& \psi(x, 0)=\psi_{0}, w(x, 0)=w_{0}, \quad x \in \Omega
\end{aligned}
$$

where $\Omega$ is a bounded domain of $\mathbb{R}^{n}$ with smooth boundary $\partial \Omega, f_{i}(i=1,2)$ are smooth real functions (let us say $C^{2}$ functions) and $g$ is a control in $L^{2}\left(Q_{T}\right)$. Let $g^{*}$ in $L^{2}\left(Q_{T}\right)$ (with $\left.Q_{T}=\Omega \times\right] 0, T[$ ), and $\left(\psi_{0}^{*}, w_{0}^{*}\right) \in L^{2}(\Omega)^{2}$. Suppose that there exists a $\left(\psi^{*}, w^{*}\right)$ satisfying $(1.1)$ in $\left.\left.C(] 0, T\right] \times L^{2}(\Omega)\right)^{2}$ with $(\psi(0), w(0))=\left(\psi_{0}^{*}, w_{0}^{*}\right)$. Therefore, by setting:

$$
\begin{aligned}
& \psi=\bar{\psi}-\psi^{*} \\
& w=\bar{w}-w^{*}
\end{aligned}
$$

where $(\bar{\psi}, \bar{w}, \bar{g})$ satisfies $(1.1)$, one gets:

$$
\begin{aligned}
& \psi_{t}=\Delta \psi+f_{1}(\bar{\psi}, \bar{w})-f_{1}\left(\psi^{*}, w^{*}\right) \text { in } Q_{T} \\
& w_{t}=\Delta w+f_{2}(\bar{\psi}, \bar{w})-f_{2}\left(\psi^{*}, w^{*}\right)+\chi_{\omega} g \text { in } Q_{T} \\
& \psi=w=0 \text { on } \Sigma_{T} \\
& \psi(x, 0)=\psi_{0}, w(x, 0)=w_{0}, x \in \Omega
\end{aligned}
$$

where $g=\bar{g}-g^{*}$. We write this last system in the following form:

$$
\begin{aligned}
& \psi_{t}=\Delta \psi+a(\psi, w) \psi+b(\psi, w) w \text { in } Q_{T} \\
& w_{t}=\Delta w+c(\psi, w) \psi+d(\psi, w) w+\chi_{\omega} g \text { in } Q_{T} \\
& \psi=w=0 \text { on } \Sigma_{T} \\
& \psi(x, 0)=\psi_{0}, w(x, 0)=w_{0}, x \in \Omega
\end{aligned}
$$

where:

$$
\begin{aligned}
& a(\psi, w)=\int_{0}^{1} \frac{\partial f_{1}}{\partial \psi}\left(s \psi+\psi^{*}, s w+w^{*}\right) d s \\
& b(\psi, w)=\int_{0}^{1} \frac{\partial f_{1}}{\partial w}\left(s \psi+\psi^{*}, s w+w^{*}\right) d s \\
& c(\psi, w)=\int_{0}^{1} \frac{\partial f_{2}}{\partial \psi}\left(s \psi+\psi^{*}, s w+w^{*}\right) d s \\
& d(\psi, w)=\int_{0}^{1} \frac{\partial f_{2}}{\partial w}\left(s \psi+\psi^{*}, s w+w^{*}\right) d s .
\end{aligned}
$$

Our aim is, for any $\left(\psi_{0}, w_{0}\right)$ belonging to a suitable space, to find a control $g \in L^{2}\left(Q_{T}\right)$ such that the associated solution of (1.3)-(1.2) satisfies

$$
\psi(T)=w(T)=0 o n \Omega
$$


This is the null-controllability or the controllability to the trajectories property.

For reaction-diffusion systems, this question has been considered in Anita-Barbu [4] with particular nonlinearities $f_{i}$. The authors proved a local exact controllability with two localized (in space) control functions (one for each equation). Another connected question is tackled by Barbu-Wang [6]. In their paper, these last authors prove, by way of direct techniques, the stabilization of system (1.1). As they pointed out, local null-controllability implies (local) stabilization but the first property is still an open problem. Our work is just concerned by this problem (nullcontrollability). We prove in this paper that, under an assumption (which does not seem very restrictive (see (4.1) below in Theorem 4), this property holds for system (1.1). Our approach is based on earlier works on the local and global null-controllability of phase-field systems and abstract parabolic-like systems (see [2], [3]).

This contribution is in keeping with the idea of controlling or stabilizing systems using the least control forces possible: works in this direction dealing with various systems governed by partial differential equations or equations in an abstract framework can be found in [1], [7].

The paper is organized as follows: We set and prove the local null-controllability of system (1.1) in the fourth section. Before this, we first prove in Section 2 a crucial observability estimate for the linearized problem(see Theorem 1 below). In the third section, we use this estimate to prove the null-controllability of a linearized system derived from (1.3).

\section{Observability estimate}

We consider in this section the problem:

$$
\begin{aligned}
& u_{t}=\Delta u+a u+b v \quad \text { in } Q_{T} \\
& v_{t}=\Delta v+c u+d v+\chi_{\omega} g \text { in } Q_{T} \\
& u=v=0 \text { on } \Sigma_{T} \\
& u(x, 0)=u_{0}, v(x, 0)=v_{0}, x \in \Omega
\end{aligned}
$$

and its adjoint problem:

$$
\begin{aligned}
& -\varphi_{t}=\Delta \varphi+a \varphi+c w \quad \text { in } Q_{T} \\
& -w_{t}=\Delta w+b \varphi+d w \quad \text { in } Q_{T} \\
& \varphi=w=0 \text { on } \Sigma_{T} \\
& \varphi(x, T)=\varphi_{0}, w(x, T)=w_{0}, x \in \Omega
\end{aligned}
$$

where $a, b, c, d \in L^{\infty}\left(Q_{T}\right)$

Following [9], let us introduce some notations. Let $\omega^{\prime} \Subset \omega$ be a subdomain of $\omega$ and let $\beta$ be a $C^{2}(\bar{\Omega})$ function such that

$$
\min \left\{|\nabla \beta(x)|, x \in \overline{\Omega \backslash \omega^{\prime}}\right\}>0 \quad \text { and } \quad \frac{\partial \beta}{\partial n} \leq 0 \text { on } \partial \Omega,
$$

where $n$ denotes the outward unit normal to $\partial \Omega$. Moreover, we can always assume that $\beta$ satisfies

$$
\min \{\beta(x), x \in \bar{\Omega}\} \geq \max \left(\frac{3}{4}\|\beta\|_{L^{\infty}(\Omega)}, \ln (3)\right) .
$$


Finally, we introduce the following functions with parameters $\lambda>0$ and $\tau>0$ :

$$
\begin{aligned}
& \rho(t, x):=e^{\lambda \beta(x)} t(T-t), \quad(t, x) \in Q_{T}, \\
& \alpha(t, x):=\tau e^{\frac{4}{3} \lambda\|\beta\|_{L^{\infty}(\Omega)}}-e^{\lambda \beta(x)} t(T-t), \quad(t, x) \in Q_{T} .
\end{aligned}
$$

Note in particular that $\rho>\frac{4}{T^{2}}$.

Then the following global Carleman estimate holds ([9. Theorem 7.1, p.288]): There exist $\lambda_{0}>0, \tau_{0}>0$ and a positive constant $C$ such that $\forall \lambda \geq \lambda_{0}, \forall \tau \geq \tau_{0}$ and $\forall s \geq-3$ the inequality

$$
\begin{aligned}
& \int_{Q_{T}}\left(\frac{1}{\lambda}\left|z_{t}\right|^{2}+\frac{1}{\lambda}\left|D_{x}^{2} z\right|^{2}+\lambda \tau^{2} \rho^{2}|\nabla z|^{2}+\lambda^{4} \tau^{4} \rho^{4} z^{2}\right) \rho^{2 s-1} e^{-2 \alpha} d x d t \\
\leq & C\left(\tau \int_{Q_{T}}\left|z_{t} \pm \Delta z\right|^{2} \rho^{2 s} e^{-2 \alpha} d x d t+\lambda^{4} \tau^{4} \int_{0}^{T} \int_{\omega^{\prime}} z^{2} \rho^{2 s+3} e^{-2 \alpha} d x d t\right),
\end{aligned}
$$

holds for any function $z$ satisfying homogeneous Dirichlet condition and such that the right-handside of (2.9) is finite. Moreover, the constants $C$ and $\lambda_{0}$ depend only on $\Omega$ and $\omega^{\prime}$. The constant $\tau_{0}$ is of the form

$$
\tau_{0}=c_{0}\left(\Omega, \omega^{\prime}\right)\left(T+T^{2}\right)
$$

The explicit dependence in time of the constants is not given in [9]. We refer to [10] where the above formula for $\tau_{0}$ is obtained.

In the sequel, the symbol $C$ will stand for various constants independent of $T$ and $a, b, c, d$.

Let us introduce the following notation: for given $\lambda$ and $\tau$, we set $\delta=\tau \rho$ and consider the functional:

$$
I(s, z)=\int_{Q_{T}}\left(\frac{1}{\lambda}\left|z_{t}\right|^{2}+\frac{1}{\lambda}|\Delta z|^{2}+\lambda \delta^{2}|\nabla z|^{2}+\lambda^{4} \delta^{4} z^{2}\right) \delta^{2 s-1} e^{-2 \alpha} d x d t
$$

On the other hand, we set:

$$
\|a, b, c, d\|_{\infty}=\left(\|a\|_{\infty}^{2}+\|b\|_{\infty}^{2}+\|c\|_{\infty}^{2}+\|d\|_{\infty}^{2}\right)^{1 / 2} .
$$

Our crucial result is the following:

Theorem 1. Let $\lambda_{0}>1, C$ being the constant given in (2.9). Assume moreover that there exists a constant $b_{0}>0$ and a domain $\omega_{b}$ such that

$$
\begin{array}{ll}
\omega_{b} \Subset \omega & \\
|b| \geq b_{0} & \omega_{b} \times\left(0, T_{0}\right)
\end{array}
$$

for some $T_{0}>0$. Then $\forall \lambda \geq \lambda_{0}, \forall \tau \geq \tau_{1}=\frac{T^{2}}{4}\left(\frac{4 C}{\lambda^{4}}\right)^{1 / 3}\|a, b, c, d\|_{\infty}^{2 / 3}, \forall s \geq-3, \forall r \in[0,2)$ there exists a constant $C=C_{r, T}$ such that:

$$
I(-3 / 2, \varphi)+I(-3 / 2, w) \leq C \int_{0}^{T} \int_{\omega} e^{-r \alpha} w^{2} d x d t
$$


PROOF. We will only sketch it. In a first step, we prove the following estimate using (2.9): $\forall \lambda \geq \lambda_{0}, \forall \tau \geq \tau_{1}=\frac{T^{2}}{4}\left(\frac{4 C}{\lambda^{4}}\right)^{1 / 3}\|a, b, c, d\|_{\infty}^{2 / 3}$ and $\forall s \geq-3$, the solution $(\varphi, w)$ of (2.3)-(2.4):

$$
I\left(-\frac{3}{2}, \varphi\right)+I\left(-\frac{3}{2}, w\right) \leq C \lambda^{4} \int_{0}^{T} \int_{\omega^{\prime}}\left(\varphi^{2}+w^{2}\right) e^{-2 \alpha} d x d t .
$$

for all $\omega^{\prime} \subset \subset \omega_{b} \subset \omega$.

This estimate already implies the null-controllability of (2.1) by two control forces (i. e. in the case where a second force $\chi_{\omega} f$ occurs in the first equation of (2.1)).

So, in a second step, we get rid of the term $\int_{0}^{T} \int_{\omega^{\prime}} \varphi^{2} e^{-2 \alpha} d x d t$ in the right-hand-side of (2.13). Actually, we introduce a suitable functional in order to estimate $\int_{0}^{T} \int_{\omega^{\prime}} \varphi^{2} e^{-2 \alpha} d x d t$ by $\int_{0}^{T} \int_{\omega} e^{-r \alpha} w^{2} d x d t$ and this will be possible for any $r \in[0,2)$. This is the main contribution of this work.

\section{Null controllability of (2.1)}

For $\varepsilon>0$ and $r \in(0,2)$, we define:

$$
J_{\varepsilon}(g)=\frac{1}{2} \int_{Q_{T}} e^{r \alpha} g^{2} d x d t+\frac{1}{2 \varepsilon}\|(u, v)(T)\|_{L^{2}(\Omega)}^{2},
$$

where $g \in L^{2}\left(Q_{T}\right)$ and $(u, v)$ is the associated solution of (2.1) with given $X_{0}=\left(u_{0}, v_{0}\right) \in H_{0}^{1}(\Omega) \times$ $H_{0}^{1}(\Omega)$. Introduce also the dual functional (see $[8]$ ):

$$
J_{\varepsilon}^{*}\left(Y_{0}\right)=\frac{1}{2} \int_{0}^{T} \int_{\omega} e^{-r \alpha} w^{2} d x d t+\frac{\varepsilon}{2}\left\|Y_{0}\right\|_{L^{2}(\Omega)}^{2}+\int_{\Omega} Y(0) \cdot X_{0} d x
$$

where $Y=(\varphi, w)$ is the solution of the backward linear system (2.3) with data $Y_{0}=\left(\varphi_{0}, w_{0}\right) \in$ $L^{2}(\Omega) \times L^{2}(\Omega)$.

By classical arguments, the minimization problems

$$
\min _{g} J_{\varepsilon}(g) \text { and } \min _{Y_{0}} J_{\varepsilon}^{*}\left(Y_{0}\right)
$$

have both exactly one solution $g_{\varepsilon}$ and $Y_{0 \varepsilon}$ respectively. Moreover by the maximum principle (or see for instance [8]) :

$$
g_{\varepsilon}=\chi_{\omega} e^{-r \alpha} w_{\varepsilon} o n Q_{T} ; Y_{0 \varepsilon}=-\frac{1}{\varepsilon}\left(u_{\varepsilon}, v_{\varepsilon}\right)(T) o n \Omega
$$

where $\left(u_{\varepsilon}, v_{\varepsilon}\right)$ (resp. $\left.\left(\varphi_{\varepsilon}, w_{\varepsilon}\right)\right)$ is the solution of (2.1) (resp. (2.3)) associated with $g_{\varepsilon}$ (resp. $\left.Y_{0 \varepsilon}\right)$. Since $J_{\varepsilon}^{*}\left(Y_{0 \varepsilon}\right) \leq 0$, we get

$$
\begin{aligned}
\frac{1}{2} \int_{0}^{T} \int_{\omega} e^{-r \alpha} w_{\varepsilon}^{2} d x d t & +\frac{1}{2 \varepsilon}\left\|\left(u_{\varepsilon}, v_{\varepsilon}\right)(T)\right\|_{L^{2}(\Omega)}^{2} \\
& \leq\left\|\left(\varphi_{\varepsilon}, w_{\varepsilon}\right)(0)\right\|_{L^{2}(\Omega)} \cdot\left\|X_{0}\right\|_{L^{2}(\Omega)}
\end{aligned}
$$

To obtain an uniform estimate, we will need the following results: 
Lemma 2. With the hypotheses of Theorem 1 for $r \in(0,2)$, any solution pair of (2.3) satisfies the estimate

$$
\|(\varphi, w)(0)\|_{L^{2}(\Omega)}^{2} \leq C_{T} \int_{0}^{T} \int_{\omega} e^{-r \alpha} w^{2} d x d t
$$

with

$$
C_{T}=\exp \left(C\left(1+\frac{1}{T}+\left(1+\|(a, b, c, d)\|_{\infty}\right) T+\|(a, b, c, d)\|_{\infty}^{4 / 3}\right)\right)
$$

where $\|(a, b, c, d)\|_{\infty}=\left(\|a\|_{\infty}^{2}+\|b\|_{\infty}^{2}+\|c\|_{\infty}^{2}+\|d\|_{\infty}^{2}\right)^{1 / 2}$.

PROOF. The proof of this lemma is by now classical and very similar to the equivalent lemma in [2]: it is essentially based on (2.12).

For $N \geq 1$, let $q_{N}$ such that

$$
\begin{gathered}
\frac{N+2}{2}<q_{N}<2 \frac{N+2}{N-2} \text { if } N \geq 3, \\
q_{N} \in(2,+\infty) \text { if } N=1,2
\end{gathered}
$$

Lemma 3. With the hypotheses of Lemma 2 for any $X_{0}=\left(u_{0}, v_{0}\right) \in\left(H_{0}^{1}(\Omega) \cap W^{2\left(1-\frac{1}{q_{N}}\right), q_{N}}(\Omega)\right)^{2}$, there exists $((u, v), g) \in\left(L^{2}\left(0, T ; H_{0}^{1}(\Omega)\right) \cap W_{q_{N}}^{2,1}\left(Q_{T}\right)\right)^{2} \times L^{q_{N}}\left(Q_{T, \omega}\right)$ satisfying (2.1) and:

$$
\begin{aligned}
(u, v)(T) & =0 \text { on } \Omega \\
\left\|\chi_{\omega} g\right\|_{L^{q_{N}}\left(Q_{T}\right)}^{2} & \leq C_{T} \quad\left\|X_{0}\right\|_{L^{2}(\Omega)}^{2}
\end{aligned}
$$

where $C_{T}$ is defined in lemma 2

ProOF. From (3.2) and (3.3), we get for all $\varepsilon>0$ :

$$
\frac{1}{2} \int_{0}^{T} \int_{\omega} e^{-r \alpha} w_{\varepsilon}^{2} d x d t+\frac{1}{2 \varepsilon}\left\|\left(u_{\varepsilon}, v_{\varepsilon}\right)(T)\right\|_{L^{2}(\Omega)}^{2} \leq C_{T}\left\|X_{0}\right\|_{L^{2}(\Omega)}^{2}
$$

We should obtain from this last estimate a control in $L^{2}\left(Q_{T}\right)$ just by passing to the limit in $\varepsilon$. But we will prove that our control is in $L^{q_{N}}\left(Q_{T}\right)$ because we will need this property in the following section. So let us introduce $\zeta_{\varepsilon}=e^{-r \alpha} w_{\varepsilon}$. It satisfies by (2.3):

$$
\begin{cases}\left(\zeta_{\varepsilon}\right)_{t}+\Delta \zeta_{\varepsilon}=f_{\varepsilon} & \text { in }(0, T) \times \Omega=Q_{T} \\ \zeta_{\varepsilon}=0 & \text { on }(0, T) \times \partial \Omega=\Sigma_{T} \\ \zeta_{\varepsilon}(T)=0 & \text { in } \Omega,\end{cases}
$$

with

$$
f_{\varepsilon}=-2 r \nabla \alpha \cdot\left(e^{-r \alpha} \nabla w_{\varepsilon}\right)+\left(\Delta\left(e^{-r \alpha}\right)+\left(e^{-r \alpha}\right)_{t}-d e^{-r \alpha}\right) w_{\varepsilon}-b e^{-r \alpha} \varphi_{\varepsilon}
$$

By parabolic regularity, we have:

$$
\left\|\zeta_{\varepsilon}\right\|_{W_{2}^{2,1}\left(Q_{T}\right)} \leq C\left\|f_{\varepsilon}\right\|_{L^{2}\left(Q_{T}\right)}
$$


On the other hand, we get easily, using (2.12) in Theorem 1 and assuming that $r \geq 1$

$$
\left\|\zeta_{\varepsilon}\right\|_{W_{2}^{2,1}\left(Q_{T}\right)}^{2} \leq C_{T} \int_{0}^{T} \int_{\omega} e^{-r \alpha} w_{\varepsilon}^{2} d x d t
$$

Now, by the embedding $W_{2}^{2,1}\left(Q_{T}\right) \hookrightarrow L^{q_{N}}\left(Q_{T}\right)$ (see for instance [11, Lemma 3.2, p. 80]):

$$
\left\|\zeta_{\varepsilon}\right\|_{L^{q_{N}}\left(Q_{T}\right)}^{2} \leq C_{T} \int_{0}^{T} \int_{\omega} e^{-r \alpha} w_{\varepsilon}^{2} d x d t
$$

Going back to our control we get

$$
\begin{aligned}
\left\|g_{\varepsilon}\right\|_{L^{q_{N}\left(Q_{T}\right)}}^{2} & =\left\|\chi_{\omega} \zeta_{\varepsilon}\right\|_{L^{q_{N}}\left(Q_{T}\right)}^{2} \\
& \leq C \int_{0}^{T} \int_{\omega} e^{-r \alpha} w_{\varepsilon}^{2} d x d t \\
& \leq C_{T}\left\|X_{0}\right\|_{L^{2}(\Omega)}^{2} .
\end{aligned}
$$

From (3.5) and [11, Theorem 10.4, p. 621], it follows, at least for a subsequence, that for $\varepsilon \rightarrow 0$ :

$$
\begin{aligned}
g_{\varepsilon} & \rightarrow g \quad \text { weakly in } L^{q_{N}}\left(Q_{T}\right) \\
\left(u_{\varepsilon}, v_{\varepsilon}\right) & \rightarrow(u, v) \text { weakly in } L^{2}\left(0, T ; H_{0}^{1}(\Omega)\right) \cap W_{q_{N}}^{2,1}\left(Q_{T}\right),
\end{aligned}
$$

and $((u, v), g)$ satisfy (2.1) with $(u, v)(T)=0$ and $\left\|\chi_{\omega} g\right\|_{L^{q_{N}\left(Q_{T}\right)}}^{2} \leq C_{T}\left\|X_{0}\right\|_{L^{2}(\Omega)}^{2}$.

\section{Local null controllability of (1.1)}

Our main result is the following:

Theorem 4. Local controllability to the trajectories: Assume that $f_{i} \in C^{2}\left(\mathbb{R}^{2}, \mathbb{R}\right)$ for $i=1,2$, and let $T>0,1 \leq N<6$. Assume also that there exists a global trajectory $\left(\psi^{*}, w^{*}, g^{*}\right)$ of $(1.1)$ such that

$$
\frac{\partial f_{1}}{\partial w}\left(\psi^{*}, w^{*}\right) \geq \mu>0 \text { a.e. on } \omega_{b} \times\left(0, T_{0}\right) \text {. }
$$

for some $0<T_{0}<T$ and $\omega_{b} \Subset \omega$. Then there is $\rho>0$ such that if $\psi_{0}, w_{0} \in H_{0}^{1}(\Omega) \cap W^{2\left(1-\frac{1}{q_{N}}\right), q_{N}}(\Omega)$ $\left(q_{N}\right.$ is defined in (3.4) ) with $\left\|\left(\psi_{0}, w_{0}\right)\right\|_{L^{\infty}(\Omega)} \leq \rho$, one can find $g \in L^{q_{N}}\left(Q_{T}\right)$ such that there exists $\left(\psi_{g}, w_{g}\right)$ solution of $(1.3)$ with $\psi_{g}, w_{g} \in W_{q_{N}}^{2,1}\left(Q_{T}\right)$ and satisfying:

$$
\psi_{g}(T)=0, w_{g}(T)=0 .
$$

Remark 5. It should be said that $\omega_{b}$ and $T_{0}$ are arbitrary in the assumption (4.1) and, so, it seems not to be a real restriction on the trajectory $\left(\psi^{*}, w^{*}, g^{*}\right)$ of $(1.1)$. For example, this hypothesis is satisfied by steady-state solutions of (1.1) if the nonlinearities $f_{1}$ and $f_{2}$ are sufficiently smooth. 
PROOF. For $R>0$, set

$$
K_{R}=\left\{(\psi, w) \in\left(L^{\infty}\left(Q_{T}\right)\right)^{2} ;\|(\psi, w)\|_{L^{\infty}\left(Q_{T}\right)}<R\right\}
$$

and consider the problem (2.1) with a fixed $(\psi, w) \in K_{R}$ in $a, b, c$ and $d$. Since $f_{i} \in C^{1}\left(R^{2}, R\right)$ and thanks to (4.1), $b$ will satisfy the assumptions of Theorem 1 for a sufficiently small $R>0$.

For each $(\psi, w) \in K_{R}$, thanks to (4.1), we apply Lemma 3 and consider the set $\digamma(\psi, w) \subset$ $L^{2}\left(Q_{T}\right)$ of all the solutions $u_{g}, v_{g} \in L^{2}\left(0, T ; H_{0}^{1}(\Omega)\right) \cap W_{q_{N}}^{2,1}\left(Q_{T}\right)$ associated with any control $g \in$ $L^{q_{N}}\left(Q_{T}\right)$ such that $\left(u_{g}, v_{g}\right)(T)=0$ a.e. $\Omega$ and $\left\|\chi_{\omega} g\right\|_{L^{q_{N}}\left(Q_{T}\right)}^{2} \leq C_{T}\left\|X_{0}\right\|_{L^{2}(\Omega)}^{2}$. The set $\digamma(\psi, w)$ is a nonempty closed convex subset of $L^{2}\left(Q_{T}\right)$. On the other hand, $\digamma\left(K_{R}\right)$ is relatively compact in $L^{2}\left(Q_{T}\right)$ and exactly as in [5], $\digamma$ is semicontinuous using [11, Theorem 10.4]. To prove that $\digamma$ has a fixed point (clearly, a fixed point of $\digamma$ is a solution of (1.3)), it remains to show that there exists $R>0$ such that $\digamma\left(K_{R}\right) \subset K_{R}$.

To do this, we first prove that:

$$
\left\|\left(u_{g}, v_{g}\right)\right\|_{L^{\infty}\left(Q_{T}\right)}^{2} \leq C_{T}\left\|X_{0}\right\|_{L^{\infty}(\Omega)}^{2} .
$$

Exactly as in [2], we get:

$$
\begin{aligned}
\left\|\left(u_{g}, v_{g}\right)(t)\right\|_{L^{\infty}(\Omega)} \leq & C\left(\left\|X_{0}\right\|_{L^{\infty}(\Omega)}+T^{-\frac{N+2}{2 q_{N}}+1}\left\|\chi_{\omega} g\right\|_{L^{q_{N}}\left(Q_{T}\right)}\right. \\
& \left.+\left(1+\|(a, b, c, d)\|_{L^{\infty}\left(Q_{T}\right)}\right) \int_{0}^{t}\left\|\left(u_{g}, v_{g}\right)_{g}(\tau)\right\|_{L^{\infty}(\Omega)} d \tau\right)
\end{aligned}
$$

and from Gronwall's inequality:

$$
\left\|\left(u_{g}, v_{g}\right)\right\|_{L^{\infty}\left(Q_{T}\right)} \leq C e^{C\left(1+\|(a, b, c, d)\|_{L^{\infty}\left(Q_{T}\right)}\right) T}\left(\left\|X_{0}\right\|_{L^{\infty}(\Omega)}+T^{-\frac{N+2}{2 q_{N}}+1}\left\|\chi_{\omega} g\right\|_{L^{q_{N}}\left(Q_{T}\right)}\right),
$$

and (4.1) follows from (4.2) and Lemma 3 .

The local controllability follows from (4.1) by taking the initial data sufficiently small.

\section{References}

[1] F. Ammar Khodja et A. Benabdallah (2000), Sufficient conditions for uniform stabilization of second order equations by dynamical controllers , Dynamics of Continuous, Discrete And Impulsive Systems, vol $7, \mathrm{n}^{\circ} 2$.

[2] F. Ammar Khodja , A. Benabdallah , C. Dupaix , I. Kostine (2002), Controllability to the trajectories of phase-field models by one control force. SIAM J. Control Optim., Vol 42 (2003) 1661-1680.

[3] F. Ammar Khodja , A. Benabdallah , C. Dupaix , I. Kostine (2004), Null-controllability of some systems of parabolic type by one control force, ESAIM: COCV (to appear).

[4] S. Anita and V. Barbu (2001), Local exact controllability of a reaction-diffusion system, Differential and Integral Equations, 14 pp. 577-587.

[5] V. Barbu (2000), Exact controllability of the superlinear heat equation, Appl. Math. Optim. 42, 73-89.

[6] V. Barbu and B. Wang (2003), Internal stabilization of semilinear parabolic systems, J. Math. Anal. Appl. 285, pp. 387-407. 
[7] A. Benabdallah and M.G. Naso (2002), Null Controllability of a Thermoelastic Plate. Abstr. Appl. Anal. 7 , no. 11, 585-599.

[8] I. Ekeland \& R. Temam (1974), Analyse convexe et problèmes variationnels, Dunod \& Gauthier-Villars.

[9] A. Fursikov (2000), Optimal control of distributed systems. Theory and applications. Translations of Mathematical Monographs, 187. American Mathematical Society, Providence, RI.

[10] E. Fernández-Cara, E Zuazua, (2000), Null and approximate controllability for weakly blowing up semilinear heat equations. Ann. Inst. H. Poincaré, Anal. Non Linéaire, 17, no. 5, 583-616.

[11] O. A. Ladyženskaja, V. A. Solonnikov and N. N. Ural'ceva (1968), Linear and Quasilinear Equations of Parabolic Type, Translations of Mathematical Monographs, Vol. 23, AMS. 